\title{
Evasion differential game with many pursuers versus one evader whose control set is a sector
}

\author{
Gafurjan Ibragimov, Idham Arif Alias*, Sharifah Anisah Syed Mafdzot \\ Universiti Putra Malaysia, 43400, Serdang, Selangor, Malaysia \\ *Corresponding author, e-mail: idham_aa@upm.edu.my
}

Received 4 Nov 2015

Accepted 8 Apr 2017

\begin{abstract}
We study a simple motion evasion differential game of many pursuers $x^{1}, \ldots, x^{m}$ and one evader $y$ in the plane. Maximum speeds of pursuers are equal to 1 , and the control set of the evader is a sector $S$ of radius greater than 1. We say that evasion is possible if $x^{i}(t) \neq y(t)$ for all $t \geqslant 0$ and $i=1, \ldots, m$. We obtain conditions that guarantee the evasion from any initial positions of the pursuers and evader.
\end{abstract}

KEYWORDS: simple motion, strategy, geometric constraint

MSC2010: 49N70 49N75 91A23

\section{INTRODUCTION}

The two-person differential game was initiated by Isaacs $^{1}$. Fundamental contributions were made in Refs. 2-9, and further new methods were developed in work such as in Refs. 10, 11.

A natural extension of a two-person differential game is a differential game with many pursuers and one evader. The latter was intensively studied in Refs. 12-15 for simple motion differential games with many pursuers. Further interesting results were obtained in Refs. 16-19.

Grigorenko $^{20}$ obtained the necessary and sufficient condition of evasion when the control sets of the players are convex compact sets. Evasion problems were studied in Ref. 21 more generally, where the game is described by

$$
\dot{z}^{i}=u^{i}-v, z^{i} \in \mathbb{R}^{k}, z^{i}(0)=z_{0}^{i}, i=1, \ldots, n,
$$

where $u^{i} \in U_{i}, v \in V$, and $U_{i}$ and $V$ are compact sets. Evasion is said to be possible if $z^{i}(t) \notin M_{i}$ for all $i=1, \ldots, n$ and $t \geqslant 0$, where $M_{i}$ for $i=1, \ldots, n$ are given nonempty convex compact sets. An evasion theorem was proved under some assumptions.

Differential games of many pursuers and one evader described by

$$
\begin{aligned}
\dot{x}^{i} & =u^{i}, x^{i}(0)=x_{0}^{i},\left|u^{i}\right| \leqslant \rho_{i}, i=1, \ldots, m, \\
\dot{y} & =v, y(0)=y_{0},|v| \leqslant \sigma
\end{aligned}
$$

where $x^{i}, y, u^{i}, v \in \mathbb{R}^{n}, \rho_{i} \geqslant 0, \sigma>0, x_{0}^{i} \neq y_{0}$, $i=1, \ldots, m$, were studied in much work. If $x^{i}(\tau)=$ $y(\tau)$ at some $i \in\{1, \ldots, m\}$ and $\tau \geqslant 0$, pursuit is said to be completed, and if $x^{i}(t) \neq y(t)$ for all $i=1, \ldots, m$, and $t \geqslant 0$, then evasion is said to be possible. Without restriction of generality, we can assume that $\sigma=1$. The following cases were studied.

Case 1. At least one of $\rho_{1}, \ldots, \rho_{m}$, e.g., $\rho_{1}$, is greater than $\sigma=1$. Then, clearly, the pursuer $x^{1}$ can complete the game. There is no difficulty in this case.

Case 2. $\rho_{i}<1$ for all $i=1, \ldots, m$. According to Ref. 13, evasion is possible in this case.

Case 3. $\rho_{1}=\cdots=\rho_{k}=1, \rho_{i}<1, i=k+$ $1, \ldots, m$. In this case, we construct the convex hull

$$
\begin{aligned}
& X=\operatorname{conv}\left\{x_{0}^{1}, \ldots, x_{0}^{k}\right\}:=\left\{\beta_{1} x_{0}^{1}+\cdots+\beta_{k} x_{0}^{k} \mid\right. \\
&\left.\beta_{1}+\cdots+\beta_{k}=1, \beta_{1} \geqslant 0, \ldots, \beta_{k} \geqslant 0\right\}
\end{aligned}
$$

of the points $x_{0}^{1}, \ldots, x_{0}^{k}$. If $y_{0} \in \operatorname{int} X$, then by Ref. 12, pursuit can be completed by the pursuers $x^{1}, \ldots, x^{k}$ in a finite time. If $y_{0} \notin X$, then by modifying the method of Refs. 12,13 , it can be easily shown that evasion is possible.

What if $y_{0}$ is on the boundary of the set $X$ ? This case has not been studied yet ${ }^{22}$. We give two examples.

Example 1 There are $m=4$ pursuers in $\mathbb{R}^{2}$ (i.e., $n=2)$.

$$
\begin{gathered}
x_{0}^{1}=(1,0), x_{0}^{2}=(-1,0), x_{0}^{3}=(0,-1), \\
x_{0}^{4}=(0,1), y_{0}=(0,0), \rho_{1}=\rho_{2}=\rho_{3}=1, \\
\rho_{4}=0, \sigma=1 .
\end{gathered}
$$




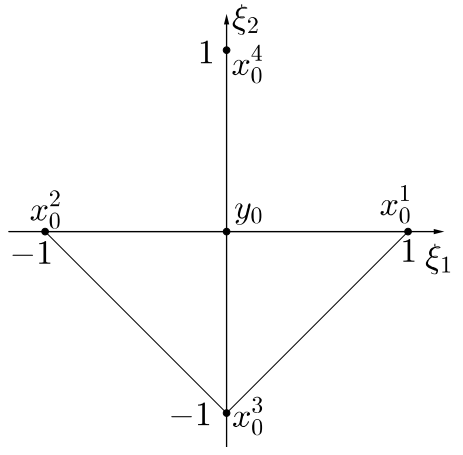

Fig. 1 The evader is on $\partial X, X \subset \mathbb{R}^{2}$.

Since $\rho_{4}=0$, the pursuer $x^{4}$ cannot move at all (Fig. 1). Nevertheless, pursuit can be completed. We give the scheme of the proof. First, set $u^{1}(t)=$ $u^{2}(t)=u^{3}(t)=(0,1), u^{4}(t)=(0,0)$. Then either $v_{2}(t)=1$ for almost all of $t \geqslant 0$, or $\left|v_{2}(t)\right|<1$ on some set $I$ with mes $I>0$. In the former case, the evader will be hit the pursuer $x^{4}$ at the time $t=1$, and hence pursuit is completed. In the latter case, $y(t) \in \operatorname{int} \operatorname{conv}\left\{x^{1}(t), x^{2}(t), x^{3}(t)\right\}$ at some $t>0$, and therefore by Ref. 12 pursuit will be completed.

In general, the case $y_{0} \in \partial X$, where $\partial X$ is the boundary of the set $X$, can be easily studied in the plane (i.e., if $n=2$ ). However, it has not been studied when $n \geqslant 3$.

Example 2 There are 9 pursuers with the initial positions $x_{0}^{1}=(-1,1,1), x_{0}^{2}=(1,1,1), x_{0}^{3}=(1,-1,1)$, $x_{0}^{4}=(-1,-1,1), x_{0}^{5}=(-1,1,-1), x_{0}^{6}=(1,1,-1)$, $x_{0}^{7}=(1,-1,-1), x_{0}^{8}=(-1,-1,-1)$, and initial position of the 9th pursuer $x_{0}^{9}$ is not specified. $\rho_{1}=$ $\cdots=\rho_{8}=1, y_{0}=(0,1,1), \sigma=1,0<\rho_{9}<1$. We can construct strategies of the pursuers $x^{1}, \ldots, x^{8}$ so that either the evader moves in the sector (Fig. 2)

$$
C=\left\{\left(\xi_{1}, \xi_{2}, \xi_{3}\right) \mid \xi_{1}=0, \xi_{2} \geqslant 1, \xi_{3} \geqslant 1\right\}
$$

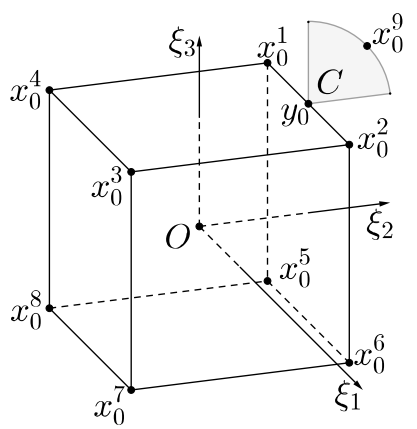

Fig. 2 The evader is on $\partial X, X \subset \mathbb{R}^{3}$. with the control $v(t), t \geqslant 0$, which must belong to the sector

$S=\left\{\left(\xi_{1}, \xi_{2}, \xi_{3}\right) \mid \xi_{1}=0, \xi_{2} \geqslant 0, \xi_{3} \geqslant 0, \xi_{2}^{2}+\xi_{3}^{2} \leqslant 1\right\}$

for almost all $t \geqslant 0$, or the inclusion

$$
y(t) \in \operatorname{int} \operatorname{conv}\left\{x^{1}(t), \ldots, x^{8}(t)\right\}
$$

holds at some $t>0$. If the inclusion (1) holds, from Ref. 12 pursuit can be completed by the pursuers $x^{1}, \ldots, x^{8}$. Hence the evader must move in the sector $C$ with a control $v(t) \in S, t \geqslant 0$. Assume that $x_{0}^{9} \in C$. The problem is to find conditions on $x_{0}^{9}$ and $\rho_{9}$ so that pursuit can be completed. It turns out that for some position $x_{0}^{9}$ and number $\rho_{9}$, pursuit can be completed.

To obtain a sufficient condition of completion of pursuit, it is reasonable to consider an auxiliary differential game of one evader $y$ and one pursuer $x^{9}$, where the control set of the evader (the set of control parameters of the evader) is the sector $S$. In the present paper, however, we will restrict the discussion to evasion games.

It should be noted that Petrov and Shchelchkov ${ }^{23}$ studied Case 3 where the definition of evasion is different from Definition 4. According to the result of that paper, in the game given in Example 1, evasion is possible.

Example 2 suggests that we should consider a differential game of many pursuers in a sector, with control set of the evader being a sector. Moreover, in this game, it is assumed that maximum speeds of the pursuers are less than that of the evader.

Simple motion differential game problems of many pursuers and one evader, where the control set of evader is a sector, can be studied independently. Hence in the present paper, we consider a simple motion differential game of many pursuers and one evader whose control set is a sector. Here, maximum speeds of the pursuers are equal to 1 and the maximum speed of the evader is $\alpha$ where $\alpha>1$, but the velocity vector of the evader belongs to the given sector. We find a sufficient condition for the evader to escape from all pursuers.

\section{STATEMENT OF THE PROBLEM AND MAIN RESULT}

We study an evasion differential game of many pursuers $x^{i}$ and one evader $y$ with geometric constraints on the controls of players in the $\left(\xi_{1}, \xi_{2}\right)$ plane. The game is described by

$$
\begin{aligned}
\dot{x}^{i} & =u^{i}, x^{i}(0)=x_{0}^{i}, i=1, \ldots, m, \\
\dot{y} & =v, y(0)=y_{0},
\end{aligned}
$$




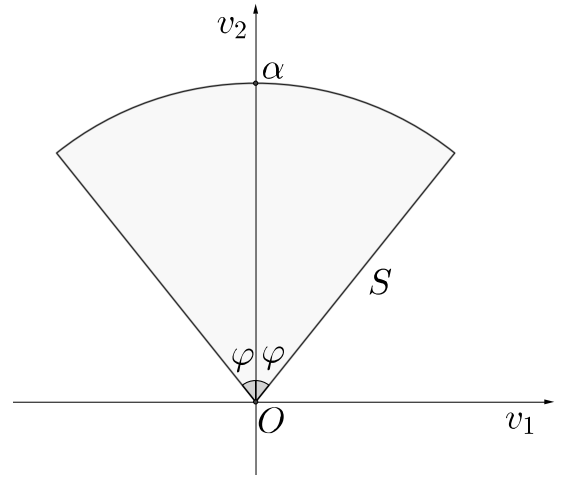

Fig. 3 Sector $S$.

where $x^{i}, x_{0}^{i}, u^{i}, y, y_{0}, v \in \mathbb{R}^{2}, x_{0}^{i} \neq y_{0},\left|u^{i}\right| \leqslant 1, v \in$ $S, u^{i}$ are the control parameters of the pursuers $x^{i}$, $v$ is that of the evader $y$,

$$
S=\left\{\left(v_{1}, v_{2}\right)\left|v_{1}^{2}+v_{2}^{2} \leqslant \alpha^{2},\right| v_{1} \mid \leqslant v_{2} \tan \varphi, v_{2} \geqslant 0\right\}
$$

is the control set of the evader, $\alpha>1$ and $\varphi$ (with $\left.0<\varphi<\frac{1}{2} \pi\right)$ is a given angle. Note that $S$ is a sector with the radius $\alpha$ and central angle $2 \varphi$ (Fig. 3).

Definition 1 A Borel measurable function, $u^{i}(t)=$ $\left(u_{1}^{i}(t), u_{2}^{i}(t)\right),\left|u^{i}(t)\right| \leqslant 1, t \geqslant 0$, is called an admissible control of the pursuer $x^{i}$.

Definition 2 A Borel measurable function, $v(t)=$ $\left(v_{1}(t), v_{2}(t)\right), v(t) \in S, t \geqslant 0$, is called an admissible control of the evader $y$.

Let $H(0, \rho)$ denote the circle of radius $\rho$ centred at the origin.

Definition 3 A Borel measurable function $V\left(t, y, x^{1}, \ldots, x^{m}, u^{1}, \ldots, u^{m}\right)$,

$$
\begin{aligned}
V:[0, \infty) \times \mathbb{R}^{2} \times \cdots & \times \mathbb{R}^{2} \\
& \times H(0, \rho) \times \cdots \times H(0, \rho) \rightarrow S,
\end{aligned}
$$

is called strategy of the evader, if for any admissible controls $u^{1}=u^{1}(t), \ldots, u^{m}=u^{m}(t)$ of pursuers the following initial value problem

$$
\begin{aligned}
\dot{x}^{1} & =u^{1}, x^{1}(0)=x_{0}^{1}, \\
& \vdots \\
\dot{x}^{m} & =u^{m}, x^{m}(0)=x_{0}^{m}, \\
\dot{y} & =V\left(t, y, x^{1}, \ldots, x^{m}, u^{1}, \ldots, u^{m}\right), y(0)=y_{0},
\end{aligned}
$$

has a unique solution $\left(x^{1}(t), \ldots, x^{m}(t), y(t)\right)$, $t \geqslant 0$ with absolutely continuous components $x^{1}(t), \ldots, x^{m}(t)$, and $y(t)$.
Definition 4 We say that evasion is possible in the game (2) if there exists a strategy $V$ of the evader $y$ such that for any admissible controls of the pursuers $x^{i}(t) \neq y(t)$ for all $t \geqslant 0$ and $i=1, \ldots, m$.

We wish to find sufficient conditions of evasion in the game (2). The condition $v(t) \in S, t \geqslant 0$, implies that the state of the evader $y(t)$ belongs to the sector $S_{1}=\left\{y_{0}+t a \mid a \in S, t \geqslant 0\right\}$. Initial positions of the pursuers may be in $S_{1}$ as well as outside $S_{1}$. In the process of pursuit, the pursuers can move throughout the plane. In this regard, the pursuers have the advantage. However, the evader has advantage of speed since $\alpha>1$.

The main result of the paper is the following statement.

Theorem 1 If $\alpha \cos \varphi_{0} \geqslant 1$ and $\alpha \sin \varphi_{0}>1$ at some $0<\varphi_{0} \leqslant \varphi$, then evasion is possible in the game (2).

To prove this theorem, first we examine a game with one pursuer. Then we show that evasion is possible in the case of many pursuers.

\section{THE CASE OF ONE PURSUER}

In this section, we consider the game with one pursuer $x^{1}$ and show that evasion is possible. Choosing any number $a_{1}$ (with $0<a_{1}<\left|x_{0}^{1}-y_{0}\right|$ ), we construct a strategy for the evader as follows:

$v(t)= \begin{cases}(0, \alpha), & 0 \leqslant t<\tau_{1}, \\ \left( \pm W_{1}^{1}(t), \sqrt{\alpha^{2}-\left(W_{1}^{1}(t)\right)^{2}}\right), & \tau_{1} \leqslant t \leqslant t_{1}, \\ (0, \alpha), & t>t_{1},\end{cases}$

where $W_{i}^{j}(s) \equiv c+\left|u_{i}^{j}(s)\right|, \quad \tau_{1}$ is the first time when $\left|y\left(\tau_{1}\right)-x^{1}\left(\tau_{1}\right)\right|=a_{1}, t_{1}=\tau_{1}+2 a_{1} / A, A=$ $\sqrt{(\alpha-1)^{2}-c^{2}}$, and $c=\alpha \sin \varphi_{0}-1$. Note that such a time $\tau_{1}$ may not exist. If so, then we let $v(t)=(0, \alpha)$ for all $t \geqslant 0$. In (3), \pm means $v_{1}(t)=$ $W_{1}^{1}(t)$, if $x_{1}^{1}\left(\tau_{1}\right) \leqslant y_{1}\left(\tau_{1}\right)$ and $v_{1}(t)=-W_{1}^{1}(t)$, if $x_{1}^{1}\left(\tau_{1}\right) \geqslant y_{1}\left(\tau_{1}\right)$, where $x^{1}(t)=\left(x_{1}^{1}(t), x_{2}^{1}(t)\right)$, $y(t)=\left(y_{1}(t), y_{2}(t)\right)$.

We estimate $\left|y(t)-x^{1}(t)\right|, \tau_{1} \leqslant t \leqslant t_{1}$. We have

$$
\begin{array}{r}
\left|y(t)-x^{1}(t)\right|=\mid y\left(\tau_{1}\right)+\int_{\tau_{1}}^{t} v(s) \mathrm{d} s \\
-\left(x^{1}\left(\tau_{1}\right)+\int_{\tau_{1}}^{t} u^{1}(s) \mathrm{d} s\right) \mid \\
\geqslant\left|y\left(\tau_{1}\right)-x^{1}\left(\tau_{1}\right)\right| \\
\quad-\left|\int_{\tau_{1}}^{t} v(s) \mathrm{d} s\right|-\left|\int_{\tau_{1}}^{t} u^{1}(s) \mathrm{d} s\right|
\end{array}
$$




$$
\geqslant a_{1}-\left(t-\tau_{1}\right)(\alpha+1) .
$$

Without loss of generality, we assume that $y_{1}\left(\tau_{1}\right) \geqslant$ $x_{1}^{1}\left(\tau_{1}\right)$, and hence $v_{1}(t)=W_{1}^{1}(t), \tau_{1} \leqslant t \leqslant t_{1}$. Then, on the other hand, for the points $x^{1}(t)$ and $y(t)$, we have

$$
\begin{gathered}
\left|y(t)-x^{1}(t)\right| \geqslant y_{1}(t)-x_{1}^{1}(t) \\
=y_{1}\left(\tau_{1}\right)+\int_{\tau_{1}}^{t} v_{1}(s) \mathrm{d} s \\
\quad-\left(x_{1}^{1}\left(\tau_{1}\right)+\int_{\tau_{1}}^{t} u_{1}^{1}(s) \mathrm{d} s\right) \\
=y_{1}\left(\tau_{1}\right)-x_{1}^{1}\left(\tau_{1}\right) \\
\quad+\int_{\tau_{1}}^{t}\left(v_{1}(s)-u_{1}^{1}(s)\right) \mathrm{d} s \\
=y_{1}\left(\tau_{1}\right)-x_{1}^{1}\left(\tau_{1}\right) \\
+\int_{\tau_{1}}^{t}\left(W_{1}^{1}(s)-u_{1}^{1}(s)\right) \mathrm{d} s \\
\geqslant c\left(t-\tau_{1}\right) .
\end{gathered}
$$

Thus $\left|y(t)-x^{1}(t)\right| \geqslant f(t)$, where $f(t)=\max \left\{a_{1}-\right.$ $\left.\left(t-\tau_{1}\right)(\alpha+1), c\left(t-\tau_{1}\right)\right\}$. Note that the function $f(t)$ has only one minimum on $\left[\tau_{1}, t_{1}\right]$ since the first argument in the max function decreases, whereas the second argument increases. The function $f(t)$ takes its minimum at

$$
t_{*}=\tau_{1}+\frac{a_{1}}{\alpha\left(1+\sin \varphi_{0}\right)} \in\left[\tau_{1}, t_{1}\right]
$$

We have

$$
\begin{aligned}
\left|y(t)-x^{1}(t)\right| & \geqslant c\left(t_{*}-\tau_{1}\right) \\
& =\frac{c a_{1}}{\alpha\left(1+\sin \varphi_{0}\right)} \\
& \geqslant \frac{c a_{1}}{2 \alpha}, \quad \tau_{1} \leqslant t \leqslant t_{1} .
\end{aligned}
$$

In particular, at the time $t_{1}$

$$
\left|y\left(t_{1}\right)-x^{1}\left(t_{1}\right)\right| \geqslant \frac{c a_{1}}{2 \alpha} .
$$

Moreover, at the time $t=t_{1}$, the pursuer cannot be above the horizontal line $\xi_{2}=y_{2}\left(t_{1}\right)$ of the $\left(\xi_{1}, \xi_{2}\right)$ plane. Indeed,

$$
\begin{aligned}
& y_{2}\left(t_{1}\right)-x_{2}^{1}\left(t_{1}\right) \\
& =y_{2}\left(\tau_{1}\right)+\int_{\tau_{1}}^{t_{1}} v_{2}(t) \mathrm{d} t \\
& \quad-\left(x_{2}^{1}\left(\tau_{1}\right)+\int_{\tau_{1}}^{t_{1}} u_{2}^{1}(t) \mathrm{d} t\right)
\end{aligned}
$$

$$
\begin{aligned}
& =y_{2}\left(\tau_{1}\right)-x_{2}^{1}\left(\tau_{1}\right) \\
& +\int_{\tau_{1}}^{t_{1}}\left(v_{2}(t)-u_{2}^{1}(t)\right) \mathrm{d} t \\
& \geqslant-a_{1}+\int_{\tau_{1}}^{t_{1}}\left(\sqrt{\alpha^{2}-\left(W_{1}^{1}(t)\right)^{2}}\right. \\
& \left.-\sqrt{1-\left|u_{1}^{1}(t)\right|^{2}}\right) \mathrm{d} t .
\end{aligned}
$$

It is not difficult to show that

$$
\sqrt{\alpha^{2}-\left(W_{1}^{1}(t)\right)^{2}}-\sqrt{1-\left|u_{1}^{1}(t)\right|^{2}} \geqslant A .
$$

Then the right-hand side of (6) can be estimated from below by

$$
-a_{1}+\int_{\tau_{1}}^{t_{1}} A \mathrm{~d} t=-a_{1}+A\left(t_{1}-\tau_{1}\right)=a_{1}>0 .
$$

Thus $y_{2}\left(t_{1}\right)-x_{2}^{1}\left(t_{1}\right) \geqslant a_{1}$. Next, according to (3), $v(t)=(0, \alpha), t>t_{1}$. Then, for $t>t_{1}$,

$$
\begin{aligned}
& y_{2}(t)-x_{2}^{1}(t) \\
& \quad=y_{2}\left(t_{1}\right)-x_{2}^{1}\left(t_{1}\right)+\int_{t_{1}}^{t}\left(\alpha-u_{2}^{1}(s)\right) \mathrm{d} s \\
& \quad \geqslant a_{1}+(\alpha-1)\left(t-t_{1}\right)>0 .
\end{aligned}
$$

In summary, for $0 \leqslant t<\tau_{1}$, by definition of $\tau_{1}$,

$$
\left|y(t)-x^{1}(t)\right|>a_{1},
$$

for $\tau_{1} \leqslant t \leqslant t_{1}$, and by (4),

$$
\left|y(t)-x^{1}(t)\right| \geqslant \frac{c a_{1}}{2 \alpha}
$$

and, for $t \geqslant t_{1}$,

$$
y_{2}(t)-x_{2}^{1}(t) \geqslant a_{1}+(\alpha-1)\left(t-t_{1}\right),
$$

from which we conclude that $y(t) \neq x^{1}(t)$ for all $t \geqslant 0$.

\section{EVASION FROM $\boldsymbol{m}$ PURSUERS}

In this section, we study the main problem, the evasion differential game of one evader from many pursuers and prove Theorem 1.

Choose a positive number $a_{1}, \quad 0<a_{1}<$ $\min _{i=1, \ldots, m}\left|x_{0}^{i}-y_{0}\right|$. Let $a_{i}=a_{1} q^{i-1}$, later. We assume that $\tau_{i}$ is the first time when a pursuer $x^{j}$ comes to within a distance $a_{i}$ of the evader $y$, i.e., $\left|x^{j}(t)-y(t)\right|>a_{i}$ for $t<\tau_{i}$, and $\left|x^{j}\left(\tau_{i}\right)-y\left(\tau_{i}\right)\right|=$ $a_{i}$. 
If there are more than one such pursuer, we take any one of them as $x^{j}$. For convenience, we denote the pursuer, whose distance from $y\left(\tau_{i}\right)$ is $a_{i}$, by $x^{i}$.

We will establish that if the $a_{i}$-approach occurs with a pursuer at some time $\tau_{i}$, then this pursuer will not participate in future $a_{j}$-approaches with $j>$ $i$ on $\left[t_{i}, \infty\right)$ and we consider this pursuer inactive starting from $t_{i}$. Thus there are given numbers $a_{1}, a_{2}, \ldots, a_{m}$ with $a_{i}=a_{1} q^{i-1}, i=1, \ldots, m$. If an $a_{i-}$ approach occurs at some time with a pursuer, then this time is denoted by $\tau_{i}$, and the pursuer is $x^{i}$ (with $i \in\{1,2, \ldots, m\}$ ).

\section{The construction of strategies for the evader and fictitious evaders}

Let $t_{i}=\tau_{i}+2 a_{i} / A$ and $V^{i}(t)=\left(V_{1}^{i}(t), V_{2}^{i}(t)\right), i=$ $1, \ldots, m$, where

$$
\begin{aligned}
V_{1}^{i}(t) & = \begin{cases}W_{1}^{i}(t), & y_{1}\left(\tau_{i}\right) \geqslant x_{1}^{i}\left(\tau_{i}\right), \\
-W_{1}^{i}(t), & y_{1}\left(\tau_{i}\right)<x_{1}^{i}\left(\tau_{i}\right),\end{cases} \\
V_{2}^{i}(t) & =\sqrt{\alpha^{2}-\left(W_{1}^{i}(t)\right)^{2}} .
\end{aligned}
$$

We call $V^{i}(t)$ a manoeuvre against the pursuer $x^{i}$. Note that $\left(V_{1}^{i}(t)\right)^{2}+\left(V_{2}^{i}(t)\right)^{2}=\alpha^{2}$, and $\left|V_{1}^{i}(t)\right| \leqslant$ $V_{2}^{i}(t) \tan \varphi_{0}$, that is, $V^{i}(t) \in S$.

We now construct a strategy for the evader. Set

$$
v(t)=(0, \alpha), 0 \leqslant t \leqslant \tau_{1},
$$

where $\tau_{1}$ is the time of the $a_{1}$-approach with the pursuer $x^{1}$. On the time interval $\left[\tau_{1}, t_{1}\right)$, the evader uses a manoeuvre against the pursuer $x^{1}$, provided an $a_{2}$-approach does not occur on this interval. Should an $a_{2}$-approach occur at some $\tau_{2} \in\left[\tau_{1}, t_{1}\right)$, the evader uses a manoeuvre against the pursuer $x^{2}$ on $\left[\tau_{2}, t_{2}\right)$ provided an $a_{3}$-approach does not occur on this interval and so on. We construct the evader's strategy precisely. The numbers $\tau_{1}, \ldots, \tau_{m}$, $t_{1}, \ldots, t_{m}$ divide the interval $\left[\tau_{1}, \infty\right)$ into disjunct intervals of the form

$$
\left[\tau_{i}, \tau_{i+1}\right),\left[\tau_{i}, t_{j}\right),\left[t_{i}, \tau_{j}\right),\left[t_{i}, t_{j}\right),\left[t_{m}^{*}, \infty\right),
$$

where $t_{m}^{*}=\max \left\{t_{1}, \ldots, t_{m}\right\}$. Also, $\tau_{1}<\ldots<\tau_{m}$, $\tau_{i}<t_{i}, i=1, \ldots, m$. Note that intervals in (7) do not contain any of the points $\tau_{1}, \ldots, \tau_{m}, t_{1}, \ldots, t_{m}$, as an interior point.

We say that the evader undergoes a continuous attack of the pursuers $x^{1}, \ldots, x^{k}$ (with $1 \leqslant k \leqslant m$ ) on the interval $\left[\tau_{1}, t_{k}^{*}\right], t_{k}^{*}=\max \left\{t_{1}, \ldots, t_{k}\right\}$, if

(i) any $t \in\left[\tau_{1}, t_{k}^{*}\right)$ belongs to an interval $\left[\tau_{i}, t_{i}\right)$, $i \in\{1, \ldots, k\}$;

(ii) $t_{k}^{*}<\tau_{k+1}$, of course, if $k+1 \leqslant m$.
Condition (i) means that the interval $\left[\tau_{1}, t_{k}^{*}\right)$ is covered by intervals $\left[\tau_{i}, t_{i}\right), i \in\{1, \ldots, k\}$. Condition (ii) means that the interval $\left[\tau_{1}, \tau_{k+1}\right)$ is not covered by intervals $\left[\tau_{i}, t_{i}\right.$ ) (with $i \in\{1, \ldots, k\}$ ) and all the intervals $\left[\tau_{i}, t_{i}\right)$ (with, $i \in\{1, \ldots, m\}$ ). In other words, the interval $\left[t_{k}^{*}, \tau_{k+1}\right)$ is not covered. The inequality $t_{k}^{*}<\tau_{k+1}$ in Condition (ii) means that a continuous attack of the pursuers $x^{1}, \ldots, x^{k}$ has been stopped at the time $t_{k}^{*}$.

We construct a strategy for the evader during the time interval $\left[\tau_{1}, t_{k}^{*}\right)$ as follows:

$$
v(t)=V^{i}(t) \text {, if } t \in\left[\tau_{i}, \tau_{i+1}\right) \text { or } t \in\left[\tau_{i}, t_{j}\right) .
$$

That is, on the intervals of the form $\left[\tau_{i}, \tau_{i+1}\right)$, $\left[\tau_{i}, t_{j}\right)$ the evader manoeuvres against the pursuer $x^{i}$,

$$
v(t)=V^{s}(t), \text { if } t \in\left[t_{i}, \tau_{j}\right) \text { or } t \in\left[t_{i}, t_{j}\right),
$$

where $s \in\{1, \ldots, k\}$ is the greatest number for which $\left[t_{i}, \tau_{j}\right) \subset\left[\tau_{s}, t_{s}\right)$ or $\left[t_{i}, t_{j}\right) \subset\left[\tau_{s}, t_{s}\right)$.

We will show that, for any $k, 1 \leqslant k \leqslant m$, the evader can 'break out' of the continuous attack of a group of $k$ pursuers on the interval $\left[\tau_{1}, t_{k}^{*}\right)$. Starting from the time $t_{k}^{*}$ we apply the same reasoning taking $t_{k}^{*}$ as the initial time, i.e.,

$$
v(t)=(0, \alpha), \text { if } t \in\left[t_{k}^{*}, \tau_{k+1}\right) .
$$

Note that after the time $t_{k}^{*}$, an $a_{k+1}$-approach may not occur. If so,

$$
v(t)=(0, \alpha), t \geqslant t_{k}^{*} .
$$

If an $a_{k+1}$-approach occurs with the pursuer $x^{k+1}$ at some time $\tau_{k+1}>t_{k}^{*}$, then the evader has to avoid the continuous attack of another group of pursuers $x^{k+1}, x^{k+2}, \ldots$, and so on.

To estimate the distances between pursuers and evader, we introduce fictitious evaders (FEs) $z^{1}, \ldots, z^{k}$ described by

$$
\dot{z}^{i}=V^{i}, z^{i}\left(\tau_{i}\right)=y\left(\tau_{i}\right), i=1, \ldots, k .
$$

Note that the initial position of $\mathrm{FE} z^{i}$ coincides with the position of the evader at the time $\tau_{i}$. FE $z^{i}$ moves only on the time interval $\left[\tau_{i}, t_{i}\right.$ ) (Fig. 4).

\section{Proof that evasion is possible}

We estimate the distance between the evader $y(t)$ and any pursuer $x^{p}(t), p \in\{1, \ldots, k\}$, from below, assuming that the evader undergoes a 'continuous attack' of the pursuers $x^{1}, \ldots, x^{k}$. If $t \leqslant \tau_{p}$, then by the definition of the time $\tau_{p}$ we have

$$
\left|x^{p}(t)-y(t)\right| \geqslant a_{p} .
$$

Now let $\tau_{p} \leqslant t<t_{p}$. 


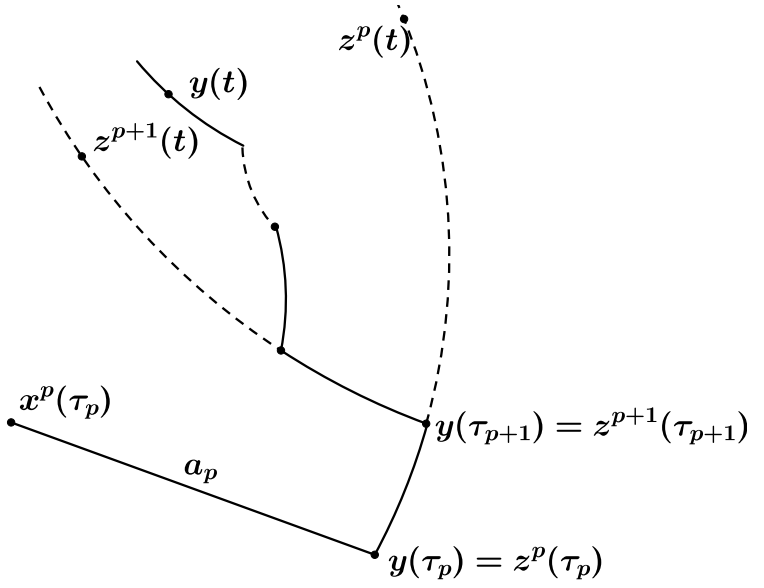

Fig. 4 Evader and FEs.

Remark 1 The interval $\left[\tau_{p}, t_{p}\right.$ ) (i) does not contain numbers $\tau_{1}, \ldots, \tau_{p-1}$ since $\tau_{1}<\ldots<\tau_{p-1}<\tau_{p}$; (ii) may contain some of the numbers $t_{1}, \ldots, t_{p-1}$; (iii) does not contain numbers $\tau_{i}, t_{i}, i \geqslant k+1$, since the evader is under continuous attack of pursuers $x_{1}, \ldots, x_{k}$, and so $t_{p} \leqslant t_{k}^{*}<\tau_{i}<t_{i}, i=k+1, \ldots$.

Remark 2 If $t_{p} \leqslant \tau_{p+1}$, then

$$
v(t)=V^{p}(t), \tau_{p} \leqslant t<t_{p} .
$$

Indeed, let

$$
\left[\tau_{p}, t_{i_{1}}\right),\left[t_{i_{1}}, t_{i_{2}}\right), \ldots,\left[t_{i_{q}}, t_{p}\right)
$$

be the disjunct subintervals of $\left[\tau_{p}, t_{p}\right)$ of the form (7) where $i_{1}, \ldots, i_{q} \in\{1,2, \ldots, p-1\}$. Then by (8)

$$
v(t)=V^{p}(t), \tau_{p} \leqslant t<t_{i_{1}},
$$

and by (9), where $s=p$,

$$
v(t)=V^{p}(t), t_{i_{r}} \leqslant t<t_{i_{r+1}},
$$

for all $r=1, \ldots, q$, where $t_{i_{q+1}}=t_{p}$.

Consider two cases: (i) an $a_{p+1}$-approach does not occur in $\left[\tau_{p}, t_{p}\right)$; (ii) an $a_{p+1}$-approach occurs in $\left[\tau_{p}, t_{p}\right)$.

Case (i). In this case $t_{p} \leqslant \tau_{p+1}$ and therefore (from Remark 2)

$$
v(t)=V^{p}(t), \tau_{p} \leqslant t<t_{p} .
$$

Then by (4) we obtain

$$
\left|x^{p}(t)-y(t)\right| \geqslant \frac{c}{2 \alpha} a_{p}, t \in\left[\tau_{p}, t_{p}\right) .
$$

Case (ii). Clearly,

$$
\left|x^{p}(t)-y(t)\right| \geqslant\left|x^{p}(t)-z^{p}(t)\right|-\left|z^{p}(t)-y(t)\right| \text {. }
$$

In view of (4),

$$
\left|x^{p}(t)-z^{p}(t)\right| \geqslant \frac{c}{2 \alpha} a_{p}, t \in\left[\tau_{p}, t_{p}\right) .
$$

Next, we estimate $\left|z^{p}(t)-y(t)\right|$. Let

$$
I=\bigcup_{i=p+1}^{k}\left[\tau_{i}, t_{i}\right)
$$

and let $\left\{\theta_{1}, \theta_{2}, \ldots, \theta_{r}\right\}, \theta_{1}<\theta_{2}<\ldots<\theta_{r}$, be the set of all elements of the set $\left\{\tau_{p}, \ldots, \tau_{k}, t_{p}, \ldots, t_{k}\right\}$ that belong to the interval $\left[\tau_{p}, t_{p}\right]$. Clearly, $\theta_{1}=\tau_{p}$, $\theta_{2}=\tau_{p+1}, \theta_{r}=t_{p}$.

$$
\begin{aligned}
& \text { Let } \\
& \begin{aligned}
L & =\left\{i \mid\left[\theta_{i}, \theta_{i+1}\right) \subset I, 1 \leqslant i \leqslant r-1\right\}, \\
M & =\left\{i \mid\left[\theta_{i}, \theta_{i+1}\right) \subset\left[\tau_{p}, t_{p}\right) \backslash I, 1 \leqslant i \leqslant r-1\right\} .
\end{aligned}
\end{aligned}
$$

Note that $L \cap M=\varnothing$ and $L \cup M=\{1,2, \ldots, r-1\}$. For example, $i=1 \in M$ since $\left[\theta_{1}, \theta_{2}\right)=\left[\tau_{p}, \tau_{p+1}\right) \subset$ $\left[\tau_{p}, t_{p}\right) \backslash I$. We show that if $i \in M$, then

$$
v(t)=V^{p}(t), \theta_{i} \leqslant t<\theta_{i+1} .
$$

Indeed, take any interval $\left[\theta_{j}, \theta_{j+1}\right), j \in M$. By Remark 1(ii), it can be divided into subintervals $J_{j 1}, \ldots, J_{j a}$ of the form (7) by some of the points $t_{1}, \ldots, t_{p-1}$ which are different from $\tau_{p}$. If $\left[\theta_{j}, \theta_{j+1}\right)$ does not contain any of the points $t_{1}, \ldots, t_{p-1}$, then put $J_{j 1}=\left[\theta_{j}, \theta_{j+1}\right)$.

As mentioned above that $\left[\theta_{1}, \theta_{2}\right)=\left[\tau_{p}, \tau_{p+1}\right)$ and by (8),

$$
v(t)=V^{p}(t), t \in J_{p 1} .
$$

Next, the relation $\left[\theta_{j}, \theta_{j+1}\right) \cap I=\varnothing$ implies that $J_{j i} \cap$ $I=\varnothing, i=1, \ldots, a$. Consequently, each set $J_{j i}, i=$ $1, \ldots, a$, is not covered by intervals $\left[\tau_{i}, t_{i}\right), i=p+$ $1, \ldots, k$. However, $J_{j i} \subset\left[\theta_{j}, \theta_{j+1}\right) \subset\left[\tau_{p}, t_{p}\right)$ for all $i=1, \ldots, a$, and therefore for each $J_{j i} \neq J_{p 1}$, by (9) we have $s=p$. Hence $v(t)=V^{p}(t), t \in J_{j i}$ and so $v(t)=V^{p}(t), t \in\left[\theta_{j}, \theta_{j+1}\right)$, which establishes (14).

To estimate $\left|z^{p}(t)-y(t)\right|$, we let $\theta_{l} \leqslant t<\theta_{l+1}$ for some $l, 1 \leqslant l \leqslant r-1$. Since $z^{p}\left(\tau_{p}\right)=y\left(\tau_{p}\right)$,

$$
\begin{aligned}
& \left|z^{p}(t)-y(t)\right| \\
& =\mid z^{p}\left(\tau_{p}\right)+\int_{\tau_{p}}^{t} V^{p}(s) \mathrm{d} s \\
& \quad-y\left(\tau_{p}\right)-\int_{\tau_{p}}^{t} v(s) \mathrm{d} s \mid \\
& \leqslant \int_{\tau_{p}}^{t}\left|V^{p}(s)-v(s)\right| \mathrm{d} s .
\end{aligned}
$$


The integral on the right-hand side of (15) can be represented as

$$
\begin{aligned}
& \sum_{i \in L, i \leqslant l-1} \int_{\theta_{i}}^{\theta_{i+1}}\left|V^{p}(s)-v(s)\right| \mathrm{d} s \\
&+\sum_{i \in M, i \leqslant l-1} \int_{\theta_{i}}^{\theta_{i+1}}\left|V^{p}(s)-v(s)\right| \mathrm{d} s \\
& \\
&+\int_{\theta_{l}}^{t}\left|V^{p}(s)-v(s)\right| \mathrm{d} s .
\end{aligned}
$$

By (14), the second sum in (16) equals 0 . If $l \in M$, then the last integral equals 0 as well. Then using the inequality $\left|V^{p}(s)-v(s)\right| \leqslant 2 \alpha$, we obtain from (15) and (16) that

$$
\begin{aligned}
\left|z^{p}(t)-y(t)\right| & \leqslant \sum_{i \in L, i \leqslant l-1} \int_{\theta_{i}}^{\theta_{i+1}} 2 \alpha \mathrm{d} s \\
& \leqslant 2 \alpha \operatorname{mes}(I) \leqslant 2 \alpha \sum_{i=p+1}^{k}\left(t_{i}-\tau_{i}\right) \\
& \leqslant 2 \alpha \sum_{i=p+1}^{\infty} \frac{2 a_{p+1}}{A}=\frac{4 \alpha a_{p} q}{A(1-q)}
\end{aligned}
$$

Let

$$
0<q<\min \left\{\frac{1}{3}, \frac{c}{4 \alpha}, \frac{A c}{A c+16 \alpha^{2}}\right\} .
$$

Substituting (13) and (17) into (12) and using (18) and $a_{p+1}=a_{p} q$ gives

$$
\begin{aligned}
\left|x^{p}(t)-y(t)\right| & \geqslant \frac{c}{2 \alpha} a_{p}-\frac{4 \alpha q}{A(1-q)} a_{p} \\
& \geqslant \frac{c}{4 \alpha} a_{p}>a_{p+1}, \tau_{p} \leqslant t \leqslant t_{p} .
\end{aligned}
$$

This inequality shows that an $a_{p+1}$-approach does not occur with the same pursuer $x^{p}$ on $\left[\tau_{p}, t_{p}\right]$. Inequalities (13) and (19) allow us to conclude that $x^{p}(t) \neq y(t), \tau_{p} \leqslant t \leqslant t_{p}$.

Now let $t \geqslant t_{p}$. We first show that $y_{2}\left(t_{p}\right)>$ $x_{2}^{p}\left(t_{p}\right)$. Indeed,

$$
\begin{aligned}
& y_{2}\left(t_{p}\right)-x_{2}^{p}\left(t_{p}\right) \\
& =y_{2}\left(\tau_{p}\right)+\int_{\tau_{p}}^{t_{p}} v_{2}(t) \mathrm{d} t-x_{2}^{p}\left(\tau_{p}\right)-\int_{\tau_{p}}^{t_{p}} u_{2}^{p}(t) \mathrm{d} t \\
& \quad \geqslant-a_{p}+\int_{\tau_{p}}^{t_{p}}\left(v_{2}(t)-u_{2}^{p}(t)\right) \mathrm{d} t \\
& \quad=-a_{p}+\left(\int_{\left[\tau_{p}, t_{p}\right] \backslash I}+\int_{\left[\tau_{p}, t_{p}\right] \cap I}\right)\left(v_{2}(t)-u_{2}^{p}(t)\right) \mathrm{d} t .
\end{aligned}
$$

Since $v_{2}(t) \geqslant \alpha \cos \varphi_{0} \geqslant 1 \geqslant u_{2}^{p}(t), t \in I$, the second integral in (20) is not negative. Then, in view of (20), we have

$$
\begin{aligned}
& y_{2}\left(t_{p}\right)-x_{2}^{p}\left(t_{p}\right) \\
& \geqslant-a_{p}+\int_{\left[\tau_{p}, t_{p}\right] \backslash I}\left(v_{2}(t)-u_{2}^{p}(t)\right) \mathrm{d} t \\
& \geqslant-a_{p}+\int_{\left[\tau_{p}, t_{p}\right] \backslash I}\left(\sqrt{\alpha^{2}-\left(W_{1}^{p}(t)\right)^{2}}\right. \\
& \quad-\sqrt{\left.1-\left|u_{1}^{p}(t)\right|^{2}\right) \mathrm{d} t} \\
& \geqslant-a_{p}+\int_{\left[\tau_{p}, t_{p}\right] \backslash I} A \mathrm{~d} t \\
& =-a_{p}+A \operatorname{mes}\left(\left[\tau_{p}, t_{p}\right] \backslash I\right) .
\end{aligned}
$$

We now estimate the measure of the set $\left[\tau_{p}, t_{p}\right] \backslash I$. By (18), $q<\frac{1}{3}$, and therefore

$$
\begin{aligned}
& \operatorname{mes}\left(\left[\tau_{p}, t_{p}\right] \backslash I\right) \geqslant \operatorname{mes}\left[\tau_{p}, t_{p}\right]-\operatorname{mes}(I) \\
& \geqslant \tau_{p}-t_{p}-\sum_{i=p+1}^{k}\left(t_{i}-\tau_{i}\right) \\
& \quad=\frac{2 a_{p}}{A}-\sum_{i=p+1}^{k} \frac{2 a_{i}}{A} \\
& >\frac{1}{A}\left(2 a_{p}-\frac{2 a_{p} q}{1-q}\right) \\
& =2 a_{p} \frac{1}{A} \frac{1-2 q}{1-q}>\frac{a_{p}}{A} .
\end{aligned}
$$

Then from (21) we obtain

$$
y_{2}\left(t_{p}\right)-x_{2}^{p}\left(t_{p}\right)>-a_{p}+\frac{A a_{p}}{A}=0 .
$$

Hence at time $t_{p}$ the evader will be above the horizontal line where the pursuer $x^{p}$ is. Thus at time $t_{p}$ the pursuer $x^{p}$ becomes 'behind' the evader. Since

$$
v_{2}(t) \geqslant \alpha \cos \varphi_{0} \geqslant 1 \geqslant u_{2}^{p}(t),
$$

then $y_{2}(t)>x_{2}^{p}(t)$ for all $t \geqslant t_{p}$.

In conclusion, each pursuer can approach within a distance $a_{p}$ of the evader not more than once. If an $a_{p}$-approach occurs with the pursuer $x^{p}$ at a time $\tau_{p}$, then the evader uses a manoeuvre on $\left[\tau_{p}, t_{p}\right]$ which ensures the inequality $y_{2}\left(t_{p}\right)>$ $x_{2}^{p}\left(t_{p}\right)$. Furthermore, the strategy of the evader guarantees him the inequality $y_{2}(t)>x_{2}^{p}(t)$ for all $t \geqslant t_{p}$. Hence the evader, starting from time $t_{p}$, will ignore the pursuer $x^{p}$, and this pursuer is no longer active. That is, we can exclude the pursuer $x^{p}$ from 
the group of pursuers at the time $t_{p}$. Thus at the time $t_{k}^{*}=\max \left\{t_{1}, \ldots, t_{k}\right\}$ all the pursuers $x^{1}, \ldots, x^{k}$ will become inactive, and we have

$$
y_{2}(t)>x_{2}^{i}(t), t \geqslant t_{k}^{*}, i=1, \ldots, k,
$$

meaning that the evader has 'broken off' the attack of the group of pursuers $x^{1}, \ldots, x^{k}$ at $t_{k}^{*}$.

\section{DISCUSSION AND CONCLUSIONS}

A simple motion evasion differential game of many pursuers and one evader whose control set is a sector has been considered in the plane. If $\alpha \cos \varphi_{0} \geqslant 1$ and $\alpha \sin \varphi_{0}>1$, at some $\varphi_{0}, 0<\varphi_{0} \leqslant \varphi$, then evasion from all pursuers has been presented. A strategy for the evader was constructed as well. Moreover, the distances between the pursuers and evader have been estimated.

The inequality $\alpha \sin \varphi_{0}>1$ at some $0<\varphi_{0} \leqslant \varphi$, in Theorem 1 is sharp. If $\alpha \sin \varphi \leqslant 1$, for example, $\alpha=5, \sin \varphi=\frac{1}{6}$, then it can be shown that the pursuer with the initial position at $(0,1)$ and speed equal to 1 can capture the evader whose initial position is $(0,0)$.

It should be noted that if the evader uses the strategy constructed in Ref. 13, of course, for a vertical line, then for some positions the condition $v(t) \in S$ fails to hold. In the present paper, we constructed a strategy for the evader, for which $v(t) \in S$ for all $t \geqslant 0$ and it guarantees the evasion.

Acknowledgements: The present research was partially supported by the National Fundamental Research Grant Scheme FRGS of Malaysia, 01-01-16-1840FR.

\section{REFERENCES}

1. Isaacs R (1965) Differential Games, Wiley, New York.

2. Blaquière A, Gerard F, Leitmann G (1969) Quantitative and Qualitative Games, Academic Press, New York.

3. Krasovskii NN, Subbotin AI (1988) Game-Theoretical Control Problems, Springer, New York.

4. Pontryagin LS (1988) Selected Works, Nauka, Moscow.

5. Petrosyan LA (1993) Differential Games of Pursuit, World Scientific, Singapore.

6. Friedman A (1971) Differential Games, Wiley, New York.

7. Hajek O (1975) Pursuit Games, Math. Sci. Engrg. Academic Press, New York.

8. Nikol'skii MS (1984) The First Direct Method of L.S. Pontryagin in Differential Games, MSU Press, Moscow.

9. Pshenichnyi BN, Ostapenko VV (1992) Differential Games, Naukova Dumka, Kiev.
10. Chikrii AA (1997) Conflict-Controlled Processes, Kluwer, Dordrecht.

11. Satimov NYu Rikhsiev BB (2000) Methods of Solving the Problem of Avoiding Encounter in Mathematical Control Theory, Fan, Tashkent.

12. Pshenichnyi BN (1976) Simple pursuit by several objects. Kibernetika 3, 145-6.

13. Chernous'ko FL (1976) A problem of evasion from many pursuers. $J$ Appl Math Mech 40, 14-24.

14. Zak VL (1978) On a problem of evading many pursuers. $J$ Appl Math 43, 456-65.

15. Blagodatskikh AI, Petrov NN (2009) Conflict Interaction Between Groups of Controlled Objects, Udmurt State Univ, Izhevsk.

16. Borowko P, Rzymowski W, Stachura A (1988) Evasion from many pursuers in the simple motion case. $J$ Math Anal Appl 135, 75-80.

17. Alexander S, Bishop R, Christ R (2009) Capture pursuit games on unbounded domain. Enseign Math 55, 1/2, 103-25.

18. Ibragimov GI, Salimi M, Amini M (2012) Evasion from many pursuers in simple motion differential game with integral constraints. Eur J Oper Res 218, 505-11.

19. Alias IA, Ibragimov GI, Rakhmanov A (2016) Evasion differential game of infinitely many evaders from infinitely many pursuers in Hilbert space. Dyn Games Appl 6, 1-13.

20. Grigorenko NL (1990) Mathematical Methods of Control of Several Dynamic Processes, MSU Press, Moscow (in Russian).

21. Chikrii AA, Prokopovich PV (1992) Simple pursuit of one evader by a group. Cybern Syst Anal 28, 438-44.

22. Azamov AA (2011) Fundamentals of Theory of Discrete Games, Niso Poligraf, Tashkent.

23. Petrov NN, Shchelchkov KA (2012) To the problem of Chernous'ko. Vestn Udmurtsk Univ Mat Mekh Komp Nauki 4, 62-7. 